\title{
Multidetector computed tomography in acute lower gastrointestinal bleeding
}

\author{
This article was published in the following Dove Press journal: \\ Reports in Medical Imaging \\ 23 November 2010 \\ Number of times this article has been viewed
}

\author{
John Palma \\ Marius Mihaila \\ Frank Pilleul \\ Département de Radiologie Digestive \\ et des Urgences, Hôpital Edouard \\ Herriot, Hospices Civils de Lyon, \\ $\mathrm{CHU}$, Lyon, France
}

Background: The aim of this study is to evaluate multidetector computed tomography (MDCT) in acute massive lower gastrointestinal bleeding, with endoscopy and surgery as reference examinations.

Methods: A single-center retrospective study involving 34 patients with acute massive lower gastrointestinal bleeding was carried out. All patients were evaluated by MDCT scan then endoscopic or surgical examinations. Sensitivity, specificity, and positive and negative predictive values of MDCT scan were calculated using the extravasation of the contrast agent as the main criterion.

Results: Extravasation of the contrast agent was found in 30 of 34 patients ( $88 \%$ ). The bleeding site seen on CT was always the same as on endoscopic or surgical examinations $(100 \%)$. Sensitivity of MDCT scan was $94 \%$, specificity $100 \%$, positive predictive value $100 \%$, and negative predictive value $50 \%(P<0.001)$. Twelve diverticulum bleedings were seen on MDCT scan compared with $13(92 \%)$ on endoscopic or surgical examinations. Angiodysplasia was overestimated by MDCT scan.

Conclusion: MDCT scan appears to be an excellent tool to find and localize the bleeding site in cases of acute massive lower gastrointestinal disease.

Keywords: MDCT, acute lower gastrointestinal bleeding, extravasation, contrast agent

\section{Introduction}

If management algorithms are relatively widely accepted for acute upper gastrointestinal (GI) bleeding, with upper endoscopy as the first test to depict and treat the bleeding site and for treatment at the same time, the management of lower GI bleeding is still controversial. ${ }^{1-3}$ The major issue, after the acute management of the bleeding, is the detection of the bleeding site. $99 \mathrm{~m}$ Tc-labeled red blood cell scintigraphy, ${ }^{4-8}$ colonoscopy, ${ }^{9-15}$ and angiography ${ }^{16-26}$ are the tests usually performed to reach this objective. ${ }^{27}$ Scintigraphy is sensitive but is of poor positive predictive value in determining the precise site of bleeding, and is also rarely available in an emergency. Colonoscopy and angiography are still controversial; they require a high level of expertise and are invasive. The potential role of multidetector computed tomography scan (MDCT scan) in acute lower GI bleeding has been emphasized recently. ${ }^{28-31}$ Nevertheless, its use in a clinical scenario remains rarely described. The purpose of our study was to illustrate the potential role of MDCT scan in acute massive lower GI bleeding experienced by a large cohort of patients, with endoscopy and surgery as reference examinations. 


\section{Material and methods}

\section{Study group}

This retrospective study was approved by the Human Research Committee of our institution in March 2007. We performed research using our clinical database records for a 51-month period (January 2005 to March 2007) that was available for retrospective review and revealed 34 patients with acute massive bleeding who underwent emergency MDCT scan. This study group included 12 women (average age: 68 years \pm 18 ) and 22 men (average age: 73 years \pm 13 ). Patient ages ranged from 23 to 95 years (mean, 71 years \pm 15 ).

\section{CT examination and interpretation of results}

MDCT scan was performed with a commercially available multidetector row CT unit (Somatom Plus 10 Volume Zoom; Siemens, Erlangen, Germany) by using the following scanning parameters: $10 \times 2.5 \mathrm{~mm}$ collimation (four detector rows and 2.5-mm section thickness), $120 \mathrm{kVp}, 165 \mathrm{mAs}$, and $12.5-\mathrm{mm}$ table speed/gantry rotation. Images were reconstructed at 3-mm intervals with a b30 soft-tissue algorithm and $1.5 \mathrm{~mm}$ image reconstruction.

The average time between patients' admission in the hospital and the start of the MDCT scan was between 1 and $12 \mathrm{~h}$. One of the contraindications of contrast-enhanced MDCT is severely impaired renal function with no possibility of hemodialysis.

In our study, although the mean age was more than 70 , we had no patient with severe clinical renal dysfunction. At the start of the procedure, $120 \mathrm{~mL}$ of nonionic iodinated contrast media (CM) (Iomeron 400; Altana, Milan, Italy) was injected intravenously through a 20 -gauge cannula at a rate of $4 \mathrm{~mL} / \mathrm{s}$ using an automated power injector (Medex, Carlsbad, CA). After a nonenhanced acquisition, multidetector row scans were obtained during the arterial phase and the portal phase, to help identify active extravasation of $\mathrm{CM}$ within the bowel lumen. Both arterial and portal phases are useful to increase the sensibility in the depiction of an extravasation of the CM. The delay between the start of administrating the $\mathrm{CM}$ and the start of scanning was $30 \mathrm{sec}$ for the injected at the arterial time and $60 \mathrm{sec}$ for the portal time. Images were obtained from the dome of the liver to the lower margin of the symphysis pubis during a single breath-hold. The evaluation of MDCT scan was based on the detection, the site, and the etiology of extravasation of the iodinated CM. Extravasation was defined by the accumulation of $\mathrm{CM}$ within a digestive lumen and by it being in linear or nodular appearance. For the accuracy of
MDCT scan in depicting acute lower GI bleeding, the lower GI tract was classified according to eight anatomic locations: small bowel, cecum, right colon, transverse colon, left colon, sigmoid, rectum, and anal canal. Presence or absence of extravasation was recorded for each anatomic location in each patient. One radiologist with 6 years of experience in abdominal CT imaging was blinded from any clinical findings. Axial transverse MDCT scan images were reviewed on a workstation while using an archiving system (Impax; AGFA, Mortsel, Belgium), allowing multiplanar reconstructions. In terms of etiology, diagnosis of hemorrhagic diverticula was defined if the extravasation of CM was definitely arising from a single diverticulum. The presence of diverticulosis was insufficient for the final diagnosis. Angiodysplasia was considered if the extravasation of $\mathrm{CM}$ was arising from a segment of bowel without any thickening or irregularity. Diagnosis of tumoral bleeding was defined if the extravasation was arising from an obvious intraluminal mass. The other etiologies were classified as having various causes such as acute hemorrhagic colitis (extravasation of CM arising from a thickened segment of bowel) or postoperative bleeding (extravasation of $\mathrm{CM}$ arising from a postoperative site). In the absence of extravasation, the MDCT scans were considered negative.

\section{Reference examinations}

After the MDCT scan interpretation, patients were systematically examined either by an endoscopy (rectoscopy or colonoscopy) or by a surgery. The site and etiology of acute lower GI bleeding were identified. The lower GI tract was classified according to the same eight anatomic locations used for MDCT scan interpretations. In case of surgery, the site of the hematochezia was defined by pathological interpretation. Etiologies were classified as either diverticulosis, angiodysplasia, tumors, miscellaneous causes, or undetermined causes. The accuracy of MDCT scan in identifying the site of acute lower GI bleeding was assessed by comparing its ability to locate extravasation for each patient with that by surgery or endoscopy. Likewise, the accuracy of MDCT scan for identifying the cause of acute lower GI bleeding was also assessed by comparing it with the etiologies of bleeding identified by surgery or endoscopy (diverticulosis, angiodysplasia, tumors, various causes, and undetermined).

\section{Analysis of clinical data}

Active massive hematochezia at the time of MDCT scan noted by the physician, relapse of hematochezia, admission of the patient to intensive care unit before or after imaging, 
hemodynamic status of the patient at the time of MDCT scan, medical history, prescribed coagulation medication, red blood cell units transfused before MDCT scan, and hemoglobin rates were all retrospectively recorded for each patient. Clinical follow-up data were collected and reviewed independently from MDCT scan examinations. In all cases, the lower GI bleeding was estimated as massive by the clinicians.

\section{Statistical analysis}

Sensitivity, specificity, accuracy, and positive and negative predictive values for detecting acute lower GI bleeding with MDCT scan were calculated on the basis of a per-patient analysis in relation to the results of endoscopy or surgery. For the purposes of statistical analysis, a true-positive finding was defined when MDCT scan depicted the presence of extravasation at the same site identified by endoscopy or surgery. A false-positive finding was defined when MDCT scan showed the presence of active bleeding which was not found even in a different location, by reference examinations. A truenegative finding was defined when a bleeding focus was not identified by both MDCT scan and reference examinations. A false-negative finding was defined as the absence of active bleeding depicted on MDCT scan despite the presence of bleeding being found by reference examinations. Accuracy was defined as the ratio of the number of cases of acute lower GI bleeding that were correctly diagnosed in our study population to the total number of patients investigated. Etiologies were classified and further analyzed according to their histopathological diagnosis and/or endoscopic identification.

\section{Results}

The final diagnosis of lower GI tract bleeding $(n=34)$ was diverticulosis $(n=13)$, angiodysplasia $(n=2)$, tumors $(n=3)$, postoperative hemorrhage $(n=5)$, rectal ulcer $(n=5)$, colitis $(n=2)$, hemorrhoids $(n=1)$, nonspecific hemorrhagic changes $(\mathrm{n}=1)$, and undetermined cause $(\mathrm{n}=2)$. Table 1 summarizes the location of etiologies by surgery or endoscopy.

\section{Location-based analysis}

Arterial phase MDCT scan depicted active extravasation of $\mathrm{CM}$ in 30 of 34 patients. With MDCT scan, CM extravasation was identified in the small bowel $(n=3)$, in the cecum $(\mathrm{n}=7)$, in the right colon $(\mathrm{n}=3)$, in the transverse colon $(n=2)$, in the left colon $(n=2)$, in the sigmoid $(n=5)$, in the rectum $(n=7)$, and in the anal canal $(n=1)$ (Table 2$)$. Among these patients with CM extravasation depicted by MDCT scan, findings were then assessed by surgery or endoscopy and acute lower GI bleeding was confirmed in all
Table I Etiologies by location on reference examination

\begin{tabular}{ll}
\hline Location & Etiology \\
\hline Small bowel $(n=4)$ & Diverticula $(n=I)$ \\
& Tumor $(n=2)$ \\
& Various $(n=I)$ \\
& Postoperative \\
Cecum $(n=7)$ & Diverticula $(n=2)$ \\
& Angiodysplasia $(n=I)$ \\
& Tumor $(n=1)$ \\
& Various $(n=3)$ \\
& Postoperative \\
Right colon $(n=3)$ & Colitis \\
& Nonspecific \\
Transverse colon $(n=2)$ & Diverticula $(n=1)$ \\
& Various $(n=2)$ \\
Left colon $(n=3)$ & Postoperative \\
Sigmoid $(n=5)$ & Diverticula $(n=1)$ \\
Rectum $(n=7)$ & Angiodysplasia $(n=I)$ \\
& Diverticula $(n=3)$ \\
& Diverticula $(n=5)$ \\
Anal canal $(n=1)$ & Various $(n=7)$ \\
Undetermined $(n=2)$ & Ulcer $(n=5)$ \\
\hline & Postoperative $(n=I)$ \\
& Rectocolitis $(n=1)$ \\
& Various $(n=I)$ \\
& Hemorrhoids \\
& \\
\hline
\end{tabular}

patients. Twenty-two patients (65\%) underwent surgery, seven patients (20\%) underwent rectoscopy, and five patients (15\%) underwent a colonoscopy. In all patients who underwent rectoscopy, a positive rectal source was always identified, and the endoscopist was confident in determining the rectum as the source of bleeding.

In two patients, reference examinations revealed a bleeding site not detected with MDCT scan (false-negative MDCT scan findings). Of these patients, one presented an infra-cm GIST (gastrointestinal stromal tumor) located at the proximal ileum. The lesion was identified by MDCT scan without extravasation. Bowel resection was performed and the follow-up confirmed the role of the lesion in the active bleeding. In the other patient, arterial phase MDCT scan failed to reveal active bleeding in a diverticulum although active bleeding was identified when a colonoscopy was performed a few hours later. Overall, patient-based accuracy of MDCT scan in the detection of acute massive lower GI bleeding was $88 \%(n=30 / 34)$. The overall sensitivity, specificity, and positive and negative predictive values for the detection of hematochezia with MDCT scan were $93.8 \%$ (30 of 32), $100 \%$ ( 2 of 2), $100 \%$ (30 of 30), and $50 \%$ (2 of 4), respectively. MDCT scan had an accuracy of 100\% for locating acute massive lower GI bleeding. The site of CM 
Table 2 Etiologies by location on MDCT

\begin{tabular}{ll}
\hline Location & Etiology \\
\hline Small bowel $(n=3)$ & Diverticula $(n=1)$ \\
& Tumor $(n=I)$ \\
& Various $(n=1)$ \\
Cecum $(n=7)$ & Postoperative \\
& Diverticula $(n=2)$ \\
& Angiodysplasia $(n=3)$ \\
& Tumor $(n=I)$ \\
Right colon $(n=3)$ & Various $(n=1)$ \\
& Colitis \\
Transverse colon $(n=2)$ & Diverticula $(n=1)$ \\
& Angiodysplasia $(n=1)$ \\
Left colon $(n=2)$ & Various $(n=1)$ \\
Sigmoid $(n=5)$ & Postoperative \\
Rectum $(n=7)$ & Diverticula $(n=1)$ \\
& Angiodysplasia $(n=1)$ \\
Anal canal $(n=1)$ & Diverticula $(n=2)$ \\
& Diverticula $(n=5)$ \\
Undetermined $(n=4)$ & Angiodysplasia $(n=6)$ \\
\hline & Various $(n=1)$ \\
& Rectocolitis \\
& Various $(n=1)$ \\
& Hemorrhoids \\
& \\
&
\end{tabular}

extravasation on MDCT scan images corresponded exactly with the location of bleeding assessed by reference examinations in all patients.

\section{Etiology-based analysis}

The overall etiology-based accuracy of MDCT scan in acute lower GI bleeding was $65 \%$ (22 of 34). The MDCT scan detected 12 hemorrhagic diverticula (92\%) (Figures 1-3). The diagnosis of angiodysplasia was made in 11 cases using MDCT scan. Only two cases of angiodysplasia (Figure 4) were assessed by reference examinations (6\%). The misdiagnoses

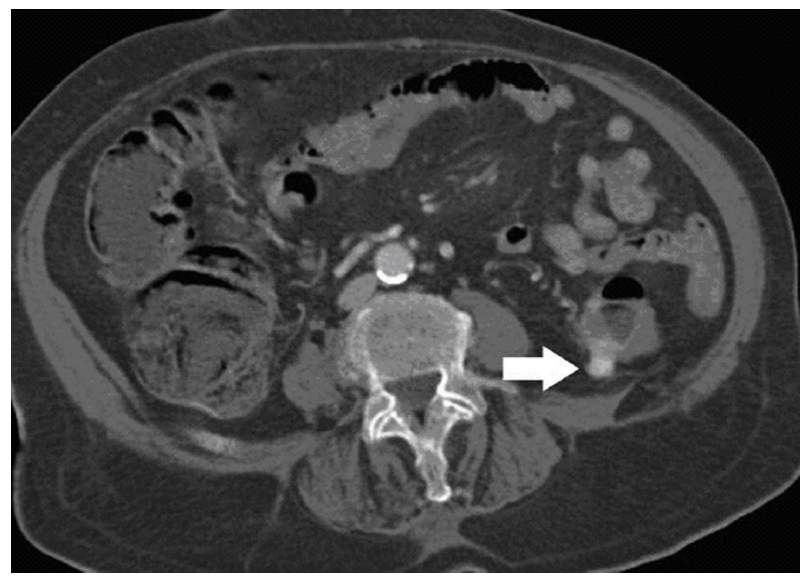

Figure I A 72-year-old male with acute lower GI bleeding. MDCT scan demonstrated an extravasation arising from a diverticulum of the left colon (arrow). Left hemicolectomy was performed for life-threatening hemorrhage.

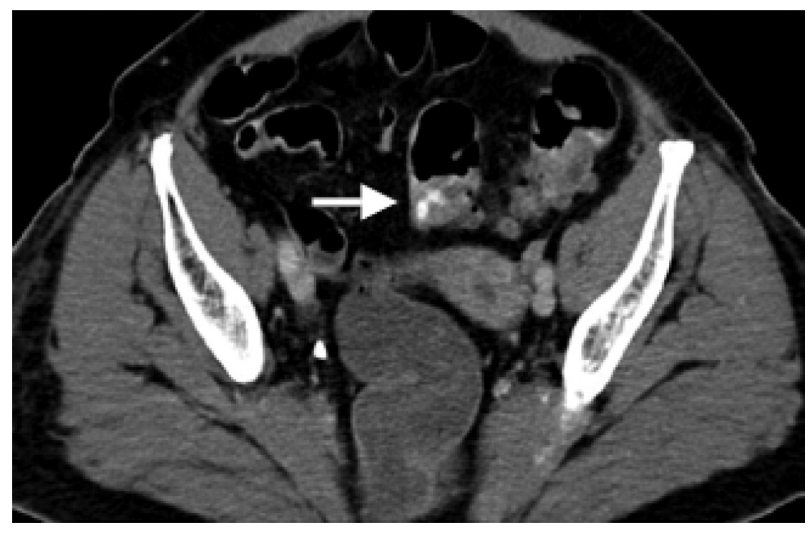

Figure 2 A 70-year-old female with acute lower Gl bleeding. MDCT scan demonstrated an extravasation arising from a diverticulum of the sigmoid (arrow). Hartman procedure was performed for life-threatening hemorrhage.

with MDCT scan were postoperative hemorrhage in three cases (Figure 5), rectal ulcer in five cases (Figure 6), and nonspecific hemorrhagic changes in one case. MDCT scan correctly identified two tumors. The missed case concerns a patient with an ileal GIST. The tumor was shown with MDCT scan, but without extravasation of CM. MDCT scan correctly identified $100 \%$ of cases of colitis $(n=2)$.

\section{Analysis of clinical data}

In 32 cases (94\%), the hematochezia were considered as active at the time of MDCT scan. In the remaining cases, the hematochezia had stopped at least $12 \mathrm{~h}$ before MDCT scan. In 32 cases (94\%), the hematochezia had relapsed. Analysis of clinical data demonstrated that bleeding was considered active in $100 \%$ of the cases with presence of extravasation and in $25 \%$ of the cases with absence of extravasation. Thus, an extravasation of CM was visible on 30 of the 31 MDCT scans performed during a

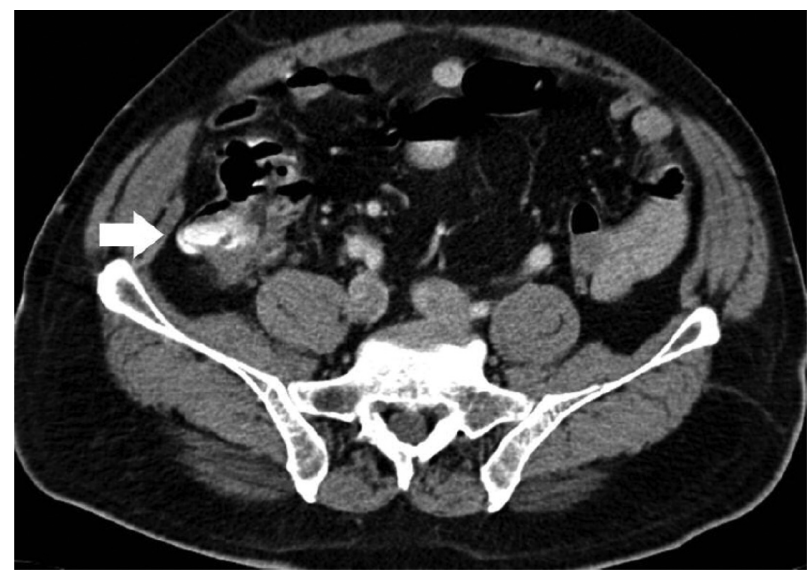

Figure 3 A 60-year-old female with acute lower Gl bleeding. MDCT scan demonstrated an extravasation arising from a diverticulum of the cecum (arrow). lleocolectomy was performed for life-threatening hemorrhage. 


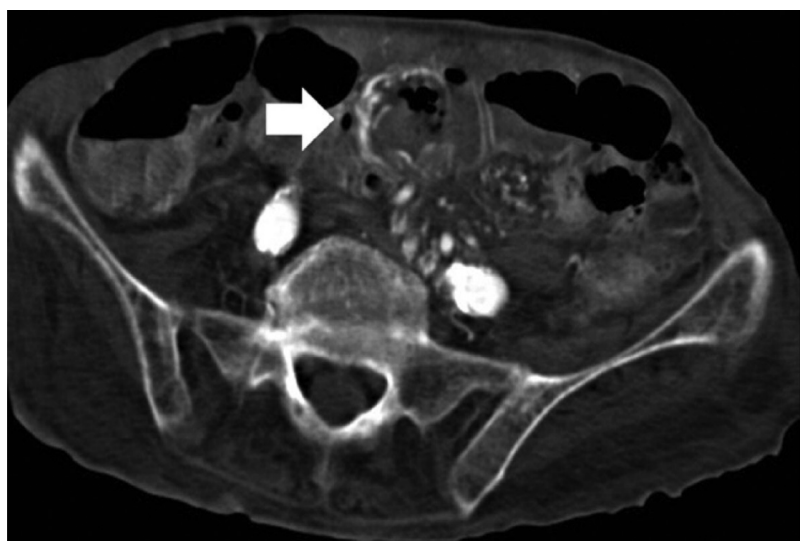

Figure 4 An 80-year-old female with acute lower Gl bleeding. MDCT scan demonstrated an extravasation arising from the transverse colon (arrow). MDCT scan was in keeping with the diagnosis of hemorrhagic angiodysplasia, which was confirmed with a colonoscopy.

hemorrhagic period (97\%). Small bowel resection occurred in four cases, ileocolectomy in four cases, right colectomy in six cases, transverse colectomy in two patients, left colectomy in three cases, Hartman procedure in three cases, endoscopic clip in three patients, and switching in five cases. Two patients died during their hospitalization. Of these two patients, one presented a hemorrhage following a polypectomy. This patient presented severe cardiac dysfunction and died from a heart attack. The second patient presented a rectal ulcer. The death was attributed to severe acute renal failure.

\section{Discussion}

Because bleeding can occur anywhere along the bowel tract, lower GI bleeding remains a clinical challenge. ${ }^{1-3}$ The major issue, after the acute management of the bleeding, is the detection of the bleeding site. Many studies discuss the

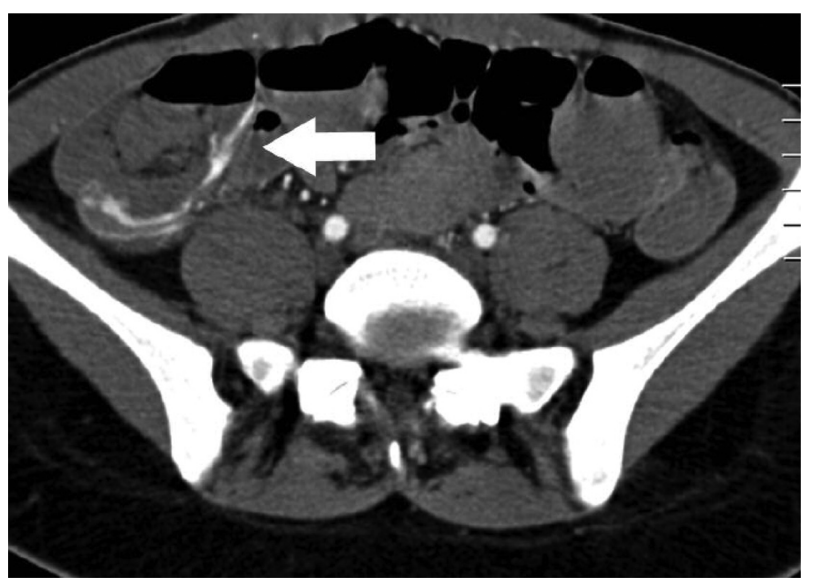

Figure 5 An 18-year-old female with acute lower Gl bleeding. MDCT scan demonstrated an extravasation arising from the cecum (arrow). MDCT scan was in keeping with the diagnosis of hemorrhagic angiodysplasia. In fact, the patient had an appendectomy 10 days prior, and an ileocolectomy was performed for lifethreatening hemorrhage.

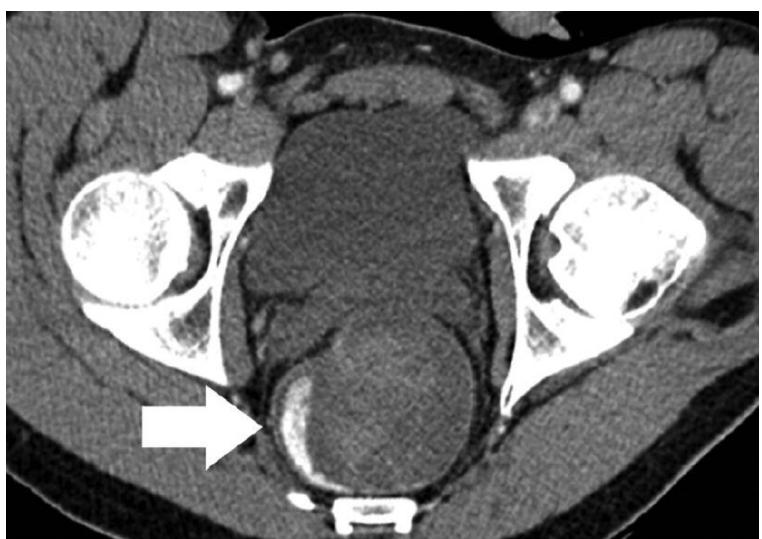

Figure 6 A 77-year-old male with acute lower GI bleeding. MDCT scan demonstrated an extravasation arising from the rectum (arrow). MDCT scan was in keeping with the diagnosis of hemorrhagic angiodysplasia. Rectoscopy demonstrated a lateral rectal ulcer, which was successfully treated at the same time.

respective role of $99 \mathrm{mTc}$-labeled red blood cell scintigraphy, ${ }^{4-8}$ colonoscopy, ${ }^{9-15}$ and angiography ${ }^{16-26}$ for that purpose.$^{27}$ The last two have the advantage of simultaneous treatment but require high expertise and are invasive, while the first is rarely available in an emergency, even if it is highly sensitive. The use of MDCT scan in acute lower GI bleeding has been rarely described in the literature. Some recent studies emphasize its possible role in the accurate detection of the bleeding site. ${ }^{28-30}$ Ettorre et al performed CT angiography with direct SMA catheterization, which is an invasive technique with good results when depicting extravasation of CM. ${ }^{32}$ Ernst et al found MDCT scan to be of intermediate sensitivity for acute lower GI bleeding, ${ }^{33}$ but their study focused on many different signs ranging from stranding to active evidence of bleeding. The prospective study by Yoon et al demonstrates high sensitivity and specificity of MDCT scan compared to angiography. ${ }^{34}$ In our series, the bleeding site found with MDCT scan was in $100 \%$ of cases concordant with the site of bleeding identified by reference examinations, with only four patients with no extravasation identified by MDCT scan. The overall patientbased accuracy of MDCT scan in detecting acute lower GI bleeding was $88 \%$, with use of CM extravasation as the only criterion for the presence of active bleeding and for locating the bleeding site, which is a good result compared to those from other retrospective studies ( 3 of 17 for Ernst et al, ${ }^{33}$ and 7 of 13 for Tew et $\left.\mathrm{al}^{35}\right)^{33-35}$ and in concordance with the prospective study by Yoon et al. ${ }^{34}$ This is also higher than what was found in the recent prospective study by Zink et al. ${ }^{31}$ A note must be made that our population presented severe bleeding at the time of MDCT scan. We are in agreement with their findings of a higher accuracy of MDCT in cases of active bleeding at the time of the acquisition. Contrary to the study by Yoon et al, our series involved a majority of patients with 
acute bleeding from diverticula, which represents the most frequent cause for recurrent hematochezia. This point is very important because the depiction of the involved diverticulum is one key in deciding the management of the bleeding, from conservative treatment to surgery in the case of ongoing lifethreatening bleeding. ${ }^{3,10,36,37}$

In our study, MDCT scan correctly identified 12 diverticulum bleedings of the 13 identified from reference examinations. Our study comprised only a small number of angiodysplasia, what can be related to that bleeding from angiodysplasia are more prone to resolve without recurrence. ${ }^{3,38-40}$ Because our study was blinded from clinical settings, the rate of angiodysplasia was overestimated. The majority of small bowel or colonic bleeding was thought to be from angiodysplasia or from postoperative bleeding. The clinical scenario would have easily corrected the diagnosis, improving the overall etiology-based accuracy of $65 \%$. MDCT scan appears as an effective tool in identifying the site of postoperative bleeding, excluding bleeding from other sources. A large number of patients in our study demonstrated bleeding from the rectum. In those cases, a rectal ulcer was the most common cause. This condition must be suggested to elderly patients with numerous comorbidities. ${ }^{41,42}$ MDCT scan enabled the elimination of other possible sites of bleeding, which provided patients with a more positive outcome after endoscopic treatment. ${ }^{42}$ Our study comprised significant limitations. Its retrospective nature limited its statistical impact. To limit recruitment bias, we included all patients with acute lower GI bleeding from our surgical emergency department who benefited from MDCT scan at their admission. The major limitation was the heterogeneity of the tools used as gold standard. Because it is a retrospective clinical study, we had no choice other than including the different examination used at the time of the clinical onset. This bias is especially important when considering a patient who only received a rectoscopy. For the seven patients who demonstrated active bleeding at the rectum and an actively bleeding rectal source at the rectoscopy, the endoscopist was confident in determining the rectum as the source of the bleeding, but another bleeding source was not completely ruled out. There is also a reading bias, because the reader was aware of the presence of intestinal bleeding. We believe that it allows a better understanding of the value of MDCT scan in this specific clinical scenario, because a reader will especially look for an active extravasation in such a case. A case-control study, although allowing stronger statistical implications, would not correspond to the reality of the radiological assessment in emergency situations. There is an evident population bias. The clinical presentation demonstrated that most patients had severe bleeding. So, we believe that our findings are only relevant for patients with massive hematochezia. This is in fact the case in almost all studies. Nevertheless, it corresponds to the correct clinical scenario, in which the correct bleeding site must be known in an emergency. Chronic or subacute bleeding is in fact not included in this discussion and is relieved of different explorations. In conclusion, our findings suggest that MDCT scan is accurate for the depiction and localization of sites of bleeding in patients with acute massive lower GI bleeding and is good at identifying the diverticulum as the source of bleeding.

\section{Disclosure}

The authors report no conflicts of interest in this work.

\section{References}

1. Farrell JJ, Friedman LS. Review article: the management of lower gastrointestinal bleeding. Aliment Pharmacol Ther. 2005;21(11):1281-1298.

2. Ghosh S, Watts D, Kinnear M. Management of gastrointestinal haemorrhage. Postgrad Med J. 2002;78(915):4-14.

3. Hoedema RE, Luchtefeld MA. The management of lower gastrointestinal hemorrhage. Dis Colon Rectum. 2005;48(11):2010-2024.

4. Bearn P, Persad R, Wilson N, Flanagan J, Williams T. 99m-Technetiumlabelled red blood cell scintigraphy as an alternative to angiography in the investigation of gastrointestinal bleeding: clinical experience in a district general hospital. Ann R Coll Surg Engl. 1992;74(3): 192-199.

5. Bentley DE, Richardson JD. The role of tagged red blood cell imaging in the localization of gastrointestinal bleeding. Arch Surg. 1991;126(7):821-824.

6. Bunker SR, Lull RJ, Tanasescu DE, et al. Scintigraphy of gastrointestinal hemorrhage: superiority of $99 \mathrm{mTc}$ red blood cells over $99 \mathrm{mTc}$ sulfur colloid. AJR Am J Roentgenol. 1984;143(3):543-548.

7. Ryan P, Styles CB, Chmiel R. Identification of the site of severe colon bleeding by technetium-labeled red-cell scan. Dis Colon Rectum. 1992; 35(3):219-222.

8. Szasz IJ, Morrison RT, Lyster DM. Technetium-99m-labeled red blood cell scanning to diagnose occult gastrointestinal bleeding. Can J Surg. $1985 ; 28(6): 512,514$

9. Jensen DM, Machicado GA. Diagnosis and treatment of severe hematochezia: the role of urgent colonoscopy after purge. Gastroenterology. 1988;95(6):1569-1574.

10. Jensen DM, Machicado GA, Jutabha R, Kovacs TO. Urgent colonoscopy for the diagnosis and treatment of severe diverticular hemorrhage. N Engl J Med. 2000;342(2):78-82.

11. Hokama A, Uehara T, Nakayoshi T, et al. Utility of endoscopic hemoclipping for colonic diverticular bleeding. Am J Gastroenterol. 1997; 92(3):543-546.

12. Green BT, Rockey DC, Portwood G, et al. Urgent colonoscopy for evaluation and management of acute lower gastrointestinal hemorrhage: a randomized controlled trial. Am J Gastroenterol. 2005; 100(11):2395-2402.

13. Chaudhry V, Hyser MJ, Gracias VH, Gau FC. Colonoscopy: the initial test for acute lower gastrointestinal bleeding. Am Surg. 1998;64(8): 723-728.

14. Caos A, Benner KG, Manier J, et al. Colonoscopy after Golytely preparation in acute rectal bleeding. J Clin Gastroenterol. 1986;8(1): 46-49.

15. Campbell WB, Rhodes M, Kettlewell MG. Colonoscopy following intraoperative lavage in the management of severe colonic bleeding. Ann R Coll Surg Engl. 1985;67(5):290-292. 
16. Dion JE, Rankin RN, Vinuela F, Fox AJ, Wallace AC, Mervart M. Dextran microsphere embolization: experimental and clinical experience with radiologic-pathologic correlation. Work in progress. Radiology. 1986;160(3):717-721.

17. Fiorito JJ, Brandt LJ, Kozicky O, Grosman IM, Sprayragen S. The diagnostic yield of superior mesenteric angiography: correlation with the pattern of gastrointestinal bleeding. Am J Gastroenterol. 1989; 84(8):878-881.

18. Luchtefeld MA, Senagore AJ, Szomstein M, Fedeson B, van Erp J, Rupp S. Evaluation of transarterial embolization for lower gastrointestinal bleeding. Dis Colon Rectum. 2000;43(4):532-534.

19. Browder W, Cerise EJ, Litwin MS. Impact of emergency angiography in massive lower gastrointestinal bleeding. Ann Surg. 1986; 204(5):530-536

20. Uden P, Jiborn H, Jonsson K. Influence of selective mesenteric arteriography on the outcome of emergency surgery for massive, lower gastrointestinal hemorrhage: a 15-year experience. Dis Colon Rectum. 1986;29(9):561-566.

21. Sos TA, Lee JG, Wixson D, Sniderman KW. Intermittent bleeding from minute to minute in acute massive gastrointestinal hemorrhage: arteriographic demonstration. AJR Am J Roentgenol. 1978;131(6): 1015-1017.

22. Molgaard CP. Mesenteric angiography for the diagnosis and treatment of lower gastrointestinal bleeding. Semin Colon Rectal Surg. 1997; 8:164-171.

23. Bloomfeld RS, Smith TP, Schneider AM, Rockey DC. Provocative angiography in patients with gastrointestinal hemorrhage of obscure origin. Am J Gastroenterol. 2000;95(10):2807-2812.

24. Walsh RM, Anain P, Geisinger M, et al. Role of angiography and embolization for massive gastroduodenal hemorrhage. J Gastrointest Surg. 1999;3(1):61-65.

25. Matolo NM, Link DP. Selective embolization for control of gastrointestinal hemorrhage. Am J Surg. 1979;138(6):840-844.

26. Kuo WT, Lee DE, Saad WE, Patel N, Sahler LG, Waldman DL. Superselective microcoil embolization for the treatment of lower gastrointestinal hemorrhage. J Vasc Interv Radiol. 2003;14(12):1503-1509.

27. Al Qahtani AR, Satin R, Stern J, Gordon PH. Investigative modalities for massive lower gastrointestinal bleeding. World J Surg. 2002;26(5): $620-625$

28. Kuhle WG, Sheiman RG. Detection of active colonic hemorrhage with use of helical CT: findings in a swine model. Radiology. 2003;228(3): 743-752.
29. Yamaguchi T, Yoshikawa K. Enhanced CT for initial localization of active lower gastrointestinal bleeding. Abdom Imaging. 2003; 28(5):634-636.

30. Krestan CR, Pokieser P, Wenzl E, Leitha T. Localization of gastrointestinal bleeding with contrast-enhanced helical CT. AJR Am J Roentgenol. 2000;174(1):265-266.

31. Zink SI, Ohki SK, Stein B, et al. Noninvasive evaluation of active lower gastrointestinal bleeding: comparison between contrast-enhanced MDCT and 99mTc-labeled RBC scintigraphy. AJR Am J Roentgenol. 2008;191(4):1107-1114.

32. Ettorre GC, Francioso G, Garribba AP, Fracella MR, Greco A, Farchi G. Helical CT angiography in gastrointestinal bleeding of obscure origin. AJR Am J Roentgenol. 1997;168(3):727-731.

33. Ernst O, Bulois P, Saint-Drenant S, Leroy C, Paris JC, Sergent G. Helical CT in acute lower gastrointestinal bleeding. Eur Radiol. 2003;13(1): 114-117.

34. Yoon W, Jeong YY, Shin SS, et al. Acute massive gastrointestinal bleeding: detection and localization with arterial phase multi-detector row helical CT. Radiology. 2006;239(1):160-167.

35. Tew K, Davies RP, Jadun CK, Kew J. MDCT of acute lower gastrointestinal bleeding. AJR Am J Roentgenol. 2004;182(2):427-430.

36. Vernava AM 3rd, Moore BA, Longo WE, Johnson FE. Lower gastrointestinal bleeding. Dis Colon Rectum. 1997;40(7):846-858.

37. Bender JS, Wiencek RG, Bouwman DL. Morbidity and mortality following total abdominal colectomy for massive lower gastrointestinal bleeding. Am Surg. 1991;57(8):536-540; discussion 540-541.

38. Breen E, Murray JJ. Pathophysiology and natural history of lower gastrointestinal bleeding. Semin Colon Rectal Surg. 1997;8:128-138.

39. Gupta N, Longo WE, Vernava AM III. Angiodysplasia of the lower gastrointestinal tract: an entity readily diagnosed by colonoscopy and primarily managed nonoperatively. Dis Colon Rectum. 1995; 38(9):979-982.

40. Baum S, Athanasoulis CA, Waltman AC, et al. Angiodysplasia of the right colon: a cause of gastrointestinal bleeding. AJR Am J Roentgenol. 1977;129(5):789-794.

41. Abdulian JD, Santoro MJ, Chen YK, Collen MJ. Dieulafoy-like lesion of the rectum presenting with exsanguinating hemorrhage: successful endoscopic sclerotherapy. Am J Gastroenterol. 1993;88(11):1939-1941.

42. Hung HY, Changchien CR, You JF, et al. Massive hematochezia from acute hemorrhagic rectal ulcer in patients with severe comorbid illness: rapid control of bleeding by per anal suturing of bleeder using anoretractor. Dis Colon Rectum. 2006;49(2):238-243.
Reports in Medical Imaging

\section{Publish your work in this journal}

Reports in Medical Imaging is an international, peer-reviewed, open access journal publishing original research, reports, reviews and commentaries on all areas of medical imaging. The manuscript management system is completely online and includes a very quick and fair peer-review system, which is all easy to use.

\section{Dovepress}

Visit http://www.dovepress.com/testimonials.php to read real quotes from published authors. 